\title{
Pharmacogenetic Testing for Methotrexate Treatment in Leukemia Patients
}

Muhammad Tahir M Bhinder, Amin Saleh Halum, Suhaib M Muflih and Mohammad Shawaqfeh ${ }^{1 *}$

College of Pharmacy, Nova Southeastern University, FL, USA

\begin{abstract}
Background: Pharmacogenetic testing can be used as a means to individualize a patient's medical regimen in order to prevent future adverse drug events. Pharmacogenomics looks at individual genes and can predetermine a patient's susceptibility to certain side effects of medications, as well as how efficacious a medication will be for that patient. Methotrexate has been shown to exhibit different responses based on the genetic expressions and variations of the genes SLC19A, SHMT, ABCB1, ATIC and MTHFR.
\end{abstract}

Objective: To determine the clinical relevance of pharamcogenetic testing for leukaemia patients treated with Methotrexate.

Method: A systematic review was conducted from September 2013-August 2015, primarily using the EMBASE and PubMed databases, identifying Cochrane reviews, controlled clinical trials, randomized control trials, meta-analyses and systematic reviews. Search terms that were initially included were the name of the genes individually (SLC19A, SHMT, ABCB1, ATIC and MTHFR), methotrexate, and leukemia. The results were further limited to English and those conducted on humans. Two reviewers extracted data and evaluated pertinent studies. A total of 82 articles were found, but were then narrowed down to 34 articles. The 34 articles were graded with the JADAD scale, with scores ranging from of 0-5 points. They were then evaluated for clinical relevance, and were reduced to 10 articles to be analysed for the purpose of the study.

Results: Of the 34 article graded, 26 articles had a score of 0 points.

Conclusion: Although there is significant evidence of an association between the clinical effects of methotrexate in leukemia patients and these genes, based on their JADAD scores, there appears to be a lack of high evidence clinical studies. None of the article found included randomized controlled trials, despite compelling evidence indicating a need for these high quality studies in order to administer methotrexate to patients in a safer manner.

Keywords: Methotrexate; Pharmacogenetic testing; Leukemia

\section{Introduction}

The ideal target of pharmacotherapy is to have optimal clinical outcomes, characterized by the complete treatment of a health illness with minimal adverse effects. As of today, is this possible? Until recently, health care providers had no idea why certain patients have a positive response to a drug therapy while others experience side effects from same therapy. It is known that patients can achieve better results when receiving individualized treatment, possibly because individualized medicine requires that one seeks a deep understanding on how medicine is metabolized by an individual's body. Although clinical practice experience indicates that to be mostly true, how can we assure the best health care of tomorrow?

A form of providing individualized care is called pharmacogenomics or pharmacogenetics. As described by the FDA, "pharmacogenomics allows one to identify sources of an individual's profile of drug response and predict the best possible treatment option for this individual. The use of genomic information has opened new possibilities in drug discovery and development." The FDA provides a table of pharmacogenomics biomarkers in drug label, in order to identify responders and nonresponders to medications, avoid adverse events, and optimize drug dose. Drug label may contain information on genomic biomarkers and can describe: drug exposure and clinical response variability, risk for adverse events, genotype specific dosing, mechanisms of drug action, and polymorphic drug target and disposition genes. For example, due to the fact that medications like codeine do not properly work for some individuals, specifically those considered ultra-rapid metabolizers for
CYP2D6; the FDA has issued boxed warnings with use for this specific population, including counseling information [1].

There are many studies proposing that, by selecting treatment based on a patient's specific genes via pharmacogenetic testing, we can predict the probability of success or failure of a particular pharmacotherapy. Based on individual specific genetic differences, pharmacogenetics can explain existing variability in drug response from its metabolism to adverse reactions. Methotrexate is commonly used as a chemotherapeutic agent for childhood acute lymphoblastic leukemia (ALL), amongst other diseases. However, treatment is limited due to severe toxicity or low efficacy of treatment for certain patients. Methotrexate has been shown to exhibit different responses based on the genetic expressions and variations of the genes SLC19A, SHMT, ABCB1, ATIC and MTHFR. For this literature evaluation, we examined the clinical relevance of pharmacogenetics testing for leukemia patients treated with Methotrexate. Furthermore, the treatment outcome for

*Corresponding author: Mohammad S Shawaqfeh, Pharm D, Ph.D, Nova Southeastern University, Palm Beach Gardens, FL, United States, Tel: 561-8052243; E-mail: mshawaqfeh@nova.edu

Received February 28, 2015; Accepted December 15, 2015; Published December 26, 2015

Citation: Bhinder MTM, Halum AS, Muflih SM, Shawaqfeh M (2015) Pharmacogenetic Testing for Methotrexate Treatment in Leukemia Patients. J Biomol Res Ther 4: 134. doi:10.4172/2167-7956.1000134

Copyright: (c) 2015 Bhinder MTM, et al. This is an open-access article distributed under the terms of the Creative Commons Attribution License, which permits unrestricted use, distribution, and reproduction in any medium, provided the original author and source are credited. 
patients with each of these genes will be taken into account as we evaluate the relevance of pharmacogenomics testing to predict these responses.

\section{Method}

In order to determine the extent to which pharmacogenetic testing could impact the clinical outcomes of leukemia patients treated with methotrexate, a literature search needed to be done. This would allow one to better understand the relationship between methotrexate toxicity and the genetic make-up of leukemia patients, in regards to the five specific genes. A systematic review (Figure 1) was conducted from September 2013-August 2015, primarily using the EMBASE and PubMed databases, identifying Cochrane reviews, controlled clinical trials, randomized control trials, meta-analyses and systematic reviews. Initially, the search terms included the name of each gene individually (SLC19A, SHMT, ABCB1, ATIC and MTHFR), methotrexate and leukemia. Two reviewers extracted data and evaluated pertinent studies. A total of 82 relevant articles were found, which were then further limited to English and those conducted on humans. The remaining articles were narrowed down, based on relevance to the study objective, leaving a total of 34 articles. The articles of interest were then graded using the JADAD grading scale to determine the strength of each article. The JADAD grading scale, which is sometimes known as JADAD scoring, is used as a grading method to define the quality of a clinical trial. Studies are individually evaluated and given a score between zeros (very poor) to five (rigorous). Since randomized control trials are considered of great importance for medical science advancement, those studies are given the highest scores. This grading criterion takes into consideration methodological errors such as selection bias and/or lack of blind-randomization, thus deducting points from those studies. Additional points are given to studies if an appropriate method of randomization or blinding was described in the paper. Sample questions for JADAD are as follows: Was the study described as randomized? Was the study described as double blind? Was there a description of withdrawals and drop-outs?

The JADAD grading scale assigns numerical points to each article based on whether it is a randomized controlled trial, if the sequence of randomization was described, if the study was double blind, a description double-blinding technique, and if there were drop-outs in the study. Based on the JADAD scores, a total of 10 articles where primarily chosen for the purpose of the study.

\section{Results}

The initial literature search, mainly using the EMBASE and PubMed databases, gave us 82 potential articles to determine the clinical relevance of pharmacogenomics testing in leukemia patients. As mentioned in the methods section, only 34 articles were used to evaluate and score using the JADAD scale. Determining the articles emphasis on the search terms also narrowed these 34 articles down. Our evaluation resulted in 26 articles with a score of zero, 5 articles with 1 point, 2 articles with 2 points and 1 article that scored 3 points. A total of 10 articles were then chosen based on the content (Table 1).

\section{Discussion}

Drug monitoring is crucial due to high variability in the pharmacokinetics of methotrexate. Methotrexate with folinic acid rescue is widely used as chemotherapy in children with acute lymphoblastic leukemia (ALL) and malignant lymphoma (ML). The different genetic expression of an individual accounts for the variability in response to treatment with methotrexate.

\section{ABCB1}

Methotrexate is a substrate for ABCB1, a member of the ATPbinding cassette transporter family. This transporter uses ATPderived energy to actively transport a variety of substrates across cell membranes. Thus, they are heavily involved in the absorption and distribution of many clinically used drugs, including anti-cancer drugs such as methotrexate (MTX) [2]. There are three single nucleotide polymorphisms (SNP's) found in the ABCB1 gene. Genetic variability can lead to the alteration of the transporter function and thus altering the sensitivity to methotrexate (MTX) treatment in children with ALL [2].

A retrospective study conducted at the University Children's Hospital Ljubljana, Slovakia, in the department of Haematology and Oncology between 1990 and 2004 concluded that the heterozygous and homozygous variant genotypes of ABCB1 2677G $>$ T/A and the ABCB1 $3435 \mathrm{C}>\mathrm{T}$ against the homozygous wild-type genotypes, and the effect of the ABCB1 T-T haplotype showed non-significant data associated with the methotrexate clearance. The results demonstrated how the SNPs do not explain substantial inter-individual variability in the systemic disposition of methotrexate [2]. However, another retrospective study at the American University of Beirut Children Cancer Center of Lebanon concluded that the polymorphisms in the ABCB1 gene showed an association with decreased exporter activity, therefore potentially leading to a slower methotrexate clearance and more toxicity. There was a strong association, specifically in neutropenia, with the ABCB1 variant allele carriers. The increase toxicity in the variant alleles

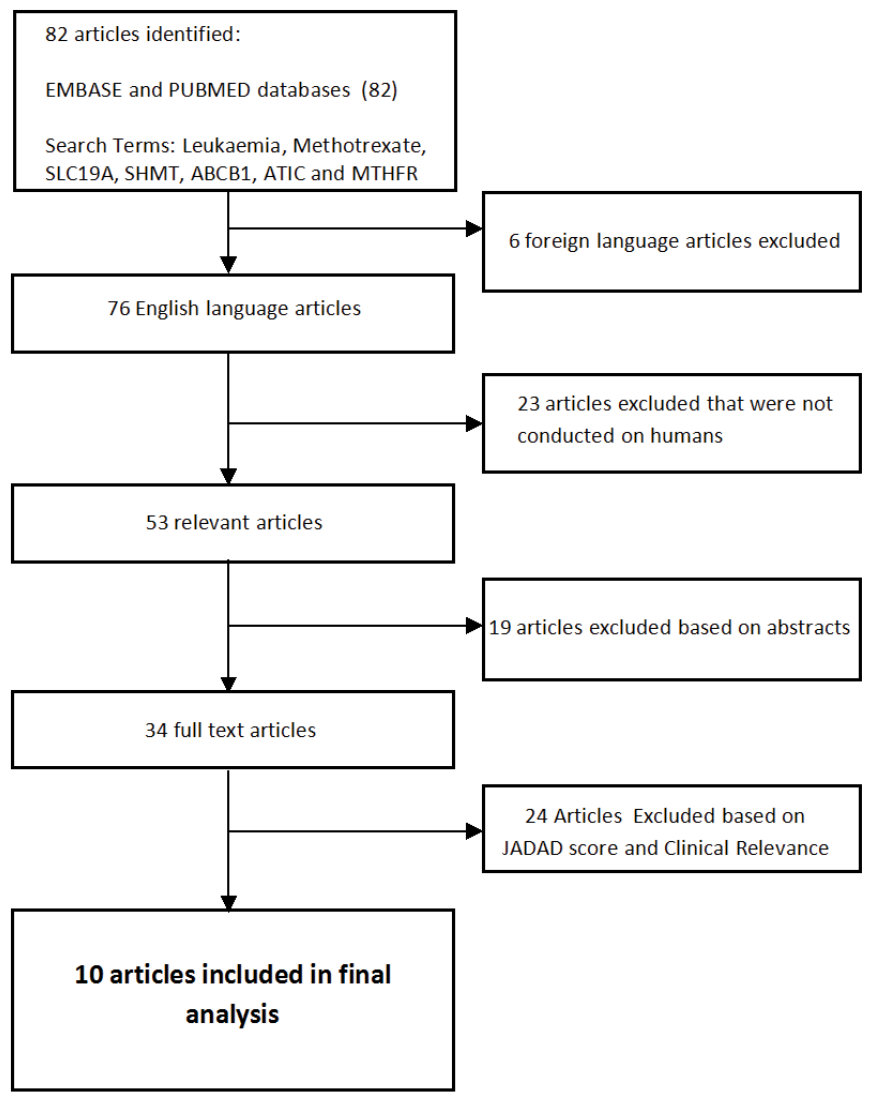

Figure 1: A systematic review was conducted from September 2013-Augus 2015, primarily using the EMBASE and PubMed databases, identifying Cochrane reviews, controlled clinical trials, randomized control trials, metaanalyses and systematic reviews. 


\section{Article Title}

Evidence Scoring

Association of genetic polymorphism in the folate metabolic pathway with methotrexate pharmacokinetics and toxicity in childhood acute lymphoblastic leukemia and malignant lymphoma ${ }^{2}$

0

Genetic polymorphisms in candidate genes predict increased toxicity with methotrexate therapy in children with acute lymphoblastic leukemia in Lebanon $^{3}$

Polyglutamation of methotrexate with common polymorphisms in reduced folate carrier, aminoimidazole carboxamide ribonucleotide transformylase, and thymidylate synthase are associated with methotrexate effects in rheumatoid arthritis ${ }^{6}$

Influence of polymorphisms within the methotrexate pathway genes on the toxicity and efficacy of methotrexate in patients with juvenile idiopathic arthritis $^{7}$

Influence of methylene tetrahydrofolate reductase C677T, A1298C, and G80A polymorphisms on the survival of pediatric patients with acute lymphoblastic leukemia ${ }^{4}$

Methotrexate toxicity and efficacy during the consolidation phase in pediatric acute lymphoblastic leukemia and MTHFR polymorphisms as pharmacogenetic determinants ${ }^{5}$

Germline genetic variations in methotrexate candidate genes are associated with pharmacokinetics, toxicity, and outcome in childhood acute lymphoblastic leukemia ${ }^{9}$

Folate pathway gene expression differs in subtypes of acute lymphoblastic leukemia and influences methotrexate pharmacodynamics ${ }^{8}$

Effect of polymorphisms in folate-related genes on in vitro methotrexate sensitivity in pediatric acute lymphoblastic leukemia ${ }^{10}$

\section{Table 1: Article and corresponding JADAD scoring.}

occurred in the consolidation phase in the ALL therapy of the Lebanese children [3]. Although theoretically ABCB1 gene polymorphism would be expected to decrease methotrexate clearance, lack of randomized controlled trials prevents us from formulating a certain conclusion.

\section{MTHFR}

5, 10-methylenetetrahydrofolate reductase, (MTHFR), is an important enzyme in the folate/methotrexate metabolism pathway. It catalyzes the conversion of 5, 10-methylenetetrahydrofolate to 5 -methyltetrahydrofolate in the folic acid cycle. Frequency of 677T, A1298C and G80A allelic variants differ in individuals and ethnicities. Differences in MTHFR activity due to these genetic variants might modify the therapeutic response to anti folate chemotherapeutic agents, including methotrexate [4].

Based on a study conducted in Hospital Oswald Cruz in Brazil, paediatric patients with 677TT genotype had a better overall survival rate than the patients with 677CC genotype for the MTHFR gene. There was a better overall survival rate for the 1298CC genotype, and although the 80AA genotype had the lowest MTX plasma level up to 24 hours, it showed a small reduction of MTX plasma concentrations in the period of 24 to 48 hours. Thus, we can suggest that patients carrying the $80 \mathrm{AA}$ genotype have difficulty in the metabolism of the chemotherapeutic agents to transport the drug into the cell, and thus, exhibit adverse effects [4]. In another study at the Paediatric Department of the Second University of Naples, the roles of the C677T and A1298C genes are compared. Two common polymorphisms, a $677 \mathrm{C}$ to $\mathrm{T}$ transition and a 1298 A to $C$ transversion, lead to $30-60 \%$ reduction in enzyme activity in the MTHFR gene. Therefore, patients with decreased MTHFR activity are at an increased risk of MTX-related toxicity. The analysis shows that patients with 677TT genotype had a 13 fold increased risk of developing non-hahematological toxicity in the 2 gram MTX dose subgroup when compared to other genotypes and doses [5].

These results contraindicate the previous study mentioned where the MTHFR C677T allele does not increase the risk of MTX induced toxicities in ALL children. This goes to show that more research needs to be done because the same alleles in a gene, such as MTHFR, show different results; one showing higher survival rate, and in another study showing a 13 fold increase risk in MTX toxicities. It could be due not only to pharmacogenetics, but also the different dosages given.

\section{AT1C}

Methotrexate enters cells through a reduced folate carrier and is activated by folypolyglutamate to MTX polyglutamate. The sequential addiction of residues enhances the intracellular retention of MTX and promotes the inhibition of 5-aminoimidazole -4- carboxamide ribonumcelotide (AICAR) transformylase (AT1C). This is the last enzyme in the purine synthesis pathway. This could lead to the accumulation of adenosine deaminase. Genetic polymorphism in the AT1C gene, among other genes, may account for part of the variability among patients in the therapeutic response to MTX [6,7]. A cross sectional study conducted at a community based Rheumatology clinic in Knoxville, TN concluded that there is a poor association between the dosage of methotrexate and its effects, but did find that patients with the AT1C C347G polymorphism did experience fewer side effects compared to patients with the AT1C 347CC or AT1C 347CG genotypes [6].

\section{SLC19, SHMT}

The reduced folate carrier SLC19 is involved with the cellular uptake of MTX. SHMT plays a role in one carbon pathway, including the conversion of tetrahydrofolate to 5,10 - methyltetrahydrofolate $[8,9]$. Different studies have been done to determine how polymorphism in these genes affects methotrexate treatment. Most studies have been inconclusive specific to these genes [10].

Overall, lack of high quality studies makes it difficult to assess the impact of polymorphism in these genes in leukemia patients taking methotrexate. The majority of the articles chosen received a score of zero on the JADAD scale, indicating a need for higher quality studies in order to be able to make a conclusion on the how pharmacogenetic testing may be able to decrease side effects and toxicity of methotrexate in leukemia patients.

\section{Limitations}

Some of the limitations that were encountered during the study were that no randomized controlled studies were found, which a gold standard trial for research studies is. Most of the articles used were on studies that had associations between the different genes, methotrexate and leukemia.

Also, two articles found results that were contraindicating to each other regarding the MTHFR gene. In one of the articles, "Methotrexate toxicity and efficacy during the consolidation phase in pediatric acute lymphoblastic leukemia and MTHFR polymorphisms as pharmacogenetic determinants", the C677T allele in the MTHFR gene had a 13-fold increase risk of developing MTX toxicity. On the 
Citation: Bhinder MTM, Halum AS, Muflih SM, Shawaqfeh M (2015) Pharmacogenetic Testing for Methotrexate Treatment in Leukemia Patients. J Biomol Res Ther 4: 134. doi:10.4172/2167-7956.1000134

Page 4 of 4

other hand, in the article, "Influence of Methylenetetrahydrofolate Reductase C677T, A1298C, and G80A Polymorphisms on the Survival of Pediatric Patients with Acute Lymphoblastic Leukemia”, the C677T allele showed it had a higher survival rate. This goes to show that more research needs to be done in patients with different genotypes as well as patients receiving different dosing of MTX. It was very difficult to find quality, evidence based research articles on the relationship between the genotypes and MTX dosing, and the side effects in leukemia patients. Another limitation that was encountered is that there were more studies on rheumatoid arthritis patients rather than leukemia patients. This was the main population that we wanted to study regarding methotrexate treatment and the responses of the different genotypes. Also, the few articles that were found about leukemia were those conducted outside of the United States in different countries such as Lebanon and China.

\section{Conclusion}

Based on this literature review, we analysed the results from our grading table and concluded that we currently lack strong evidence to support any specific pharmacogenetic testing to predict the response of methotrexate treatment in patients with childhood ALL. The main reason for this fact is that the trials were not designed to meet our current gold standard criteria of double blind randomized control trials. Because methotrexate is considered an invasive therapy with high potential for side effects and adverse reactions, it is ideal to be able to pre-test a patient to see if this therapy will potentially work or not. At this moment, it was found that there is a need of stronger evidence in order to affirm any specific pharmacogenetic testing to predict the response of methotrexate. Based on this finding, we recommend that double blind randomized control trials are designed to support stronger evidence of the effectiveness of a specific pharmacogenetic test prior to methotrexate treatment. By following this approach, we can possibly be able to provide a more individualized treatment for patients with childhood acute lymphoblastic leukemia (ALL). This will help diminish side effects, adverse reactions, and any potential health risk to the patient, making methotrexate therapy safer and more effective.

\section{References}

1. Table of Pharmacogenomic Biomarkers in Drug Labeling (2014).

2. Faganel KB, Grabnar I, Bohanec GP, Dolžan V, Jazbec J (2011) Association of genetic polymorphism in the folate metabolic pathway with methotrexate pharmacokinetics and toxicity in childhood acute lymphoblastic leukaemia and malignant lymphoma. Eur J Clin Pharmacol 67: 993-1006.

3. Zgheib N, Akra-lsmail M, Aridi C (2013) Genetic polymorphisms in candidate genes predict increased toxicity with methotrexate therapy in Lebanese children with acute lymphoblastic leukemia. Blood 24: 387-396.

4. Vasconcelos de Deus DM, Santos de Lima EI, Silva RM (2012) Influence of Methylenetetrahydrofolate Reductase C677T, A1298C, and G80A Polymorphisms on the Survival of Pediatric Patients with Acute Lymphoblastic Leukemia. Leukemia Research and Treatment 292043.

5. D'Angelo V, Ramaglia M, lannotta A, Crisci S, Indolfi P, et al. (2011) Methotrexate toxicity and efficacy during the consolidation phase in paediatric acute lymphoblastic leukaemia and MTHFR polymorphisms as pharmacogenetic determinants. Cancer Chemother Pharmacol 68: 1339-1346.

6. Dervieux T, Furst D, Lein DO, Capps R, Smith K, et al. (2004) Polyglutamation of Methotrexate with Common Polymorphisms in Reduced Folate Carrier, Aminoimidazole Carboxamide Ribonucleotide Transformylase, and Thymidylate Synthase are Associated with Methotrexate Effects in Rheumatoid Arthritis. Arthritis Rheum50: 2766-2774.

7. Yanagimachi M, Naruto T, Hara T, Kikuchi M, Hara R, et al. (2011) Influence of Polymorphisms Within the Methotrexate Pathway Genes on the Toxicity and Efficacy of Methotrexate in Patients With Juvenile Idiopathic Arthritis. $\mathrm{Br} \mathrm{J}$ Clin Pharmacol 71: 237-243.

8. Kager L, Cheok M, Yang W, Zaza G, Cheng Q, et al. (2005) Folate pathway gene expression differs in subtypes of acute lymphoblastic leukemia and influences methotrexate pharmacodynamics. J Clin Invest 115: 110-117.

9. Radtke S, Zolk O, Renner B, Paulides M, Zimmermann M, et al. (2013) Germline genetic variations in methotrexate candidate genes are associated with pharmacokinetics, toxicity, and outcome in childhood acute lymphoblastic leukemia. Blood 121: 5145-5153.

10. De Jonge $\mathrm{R}$, Hooijberg $\mathrm{JH}$, Van Zelst $\mathrm{BD}$, Jansen $\mathrm{G}$, Van Zantwijk $\mathrm{CH}$, et al. (2005) Effect of polymorphisms in folate-related genes on in vitro methotrexate sensitivity in pediatric acute lymphoblastic leukemia. Blood 106: 717-720. 